\title{
Administration effects of single-dose GnRH agonist for luteal support in females undertaking IVF/ICSI cycles: A meta-analysis of randomized controlled trials
}

\author{
MENGLING SONG ${ }^{1,2^{*}}$, CHUNLIAN LIU ${ }^{1,2^{*}}$, RONG HU $^{1,2}$, FEIMIAO WANG $^{1,2}$ and ZHENGHAO HUO ${ }^{2}$ \\ ${ }^{1}$ Department of Reproductive Medicine, General Hospital of Ningxia Medical University; \\ ${ }^{2}$ Key Laboratory of Fertility Preservation and Maintenance of Ministry of Education, Ningxia Medical University, \\ Yinchuan, Ningxia Hui Autonomous Region 750004, P.R. China
}

Received January 27, 2019; Accepted September 13, 2019

DOI: $10.3892 /$ etm.2019.8251

\begin{abstract}
The aim of the present meta-analysis was to evaluate the effects of the addition of single-dose gonadotropin-releasing hormone agonist (GnRHa) for luteal support on pregnancy outcomes in females partaking in in vitro fertilization or intracytoplasmic sperm injection cycles. In total, the studies were hand-searched from six electronic databases to compare the pregnancy outcomes between single-dose GnRHa administered as luteal phase support (GnRHa group) and regular luteal support (control group). In the GnRHa group, single-dose GnRH agonist were administered at 5/6 days after IVF/ICSI procedures. In the control group, single-dose GnRH agonist was not added during luteal phase support. Only randomized controlled trials were included. Sensitivity analysis was performed using Revman 5.3 software; the high heterogeneity identified in the present analysis was primarily caused by one study included. Following exclusion of this particular study, the meta-analysis results indicated significantly higher rates of ongoing pregnancy or live birth per transfer $(\mathrm{P}=0.002)$, clinical pregnancy per transfer $(\mathrm{CPR} ; \mathrm{P}=0.001)$ and multiple pregnancy per pregnancy $(\mathrm{P}=0.020)$ in the $\mathrm{GnRHa}$ group compared with those in the control group. Meta-analysis of a subgroup of trials with long-acting GnRH-a ovarian treatment protocols indicated that the rate of ongoing pregnancy or live birth $(\mathrm{P}=0.080), \mathrm{CPR}$ $(\mathrm{P}=0.090)$ and multiple pregnancy per pregnancy $(\mathrm{P}=0.140)$ were not significantly different between the two groups. However, the
\end{abstract}

Correspondence to: Mrs. Mengling Song, Department of Reproductive Medicine, General Hospital of Ningxia Medical University, 804 Shengli Street, Xingqing Square, Yinchuan, Ningxia Hui Autonomous Region 750004, P.R. China

E-mail: smlsong2010@163.com

${ }^{*}$ Contributed equally

Key words: gonadotropin-releasing hormone agonist, luteal phase support, in vitro fertilization/intracytoplasmic sperm injection, randomized controlled trial, meta-analysis results from trials that had used a multi-dose GnRH antagonist ovarian treatment protocol indicated a significantly higher ongoing pregnancy or live birth rate per transfer $(\mathrm{P}=0.010)$, CPR per transfer $(\mathrm{P}<0.0001)$ and multiple pregnancy rate per pregnancy $(\mathrm{P}=0.003)$ compared with those in the control group. The present results suggested that administration of single-dose GnRH agonist in the luteal phase may be an ideal choice for patients undergoing IVF/ICSI therapy.

\section{Introduction}

In the natural reproductive cycle, the luteal phase is the result of intermittent stimulation of the corpus luteum by pituitary luteinizing hormone ( $\mathrm{LH})$, which is different from assisted reproductive technology (ART) cycles. Luteal-phase deficiency is common during the follicular stimulation phase of the menstrual cycle (1) and leads to a decreased embryo implantation rate, a lower pregnancy rate and an increased miscarriage rate (2). Controlled ovarian stimulation is usually involved in in vitro fertilization (IVF)/intracytoplasmic sperm injection (ICSI)/embryo transfer (ET) technology, causing the development of multiple follicles and the formation of multiple embryos to increase the clinical pregnancy rate (CPR) and live birth rate. The development of multiple follicles leads to abnormally high levels of estrogen and multiple corpora lutea are formed after aspiration, which maintain the abnormally high production of steroids $(3,4)$. In a natural pregnancy, LH is continuously produced after the ovulation surge until human chorionic gonadotropin (hCG) is secreted by the proliferating trophoblast. In addition, the high steroid levels exert a negative feedback on the pituitary gland, thus prematurely inhibiting the production of LH during ART cycles (4). By contrast, aspiration of granulosa cells may interfere with the production of progesterone $(\mathrm{P})(5)$, leading to a reduction in the luteal phase to cause a condition known as premature luteolysis (3).

To overcome this issue, pharmacological support, including combinations of estradiol, $\mathrm{P}$ and hCG, have been frequently applied to directly or indirectly increase the low levels of $\mathrm{P}(6)$. Recently, gonadotropin-releasing hormone $(\mathrm{GnRH})$ agonist has been used for luteal-phase support (LPS) $(7,8)$. A possible explanation for the effect of GnRH agonist is that it extends 
LH production, thus preventing the occurrence of premature luteolysis (3). However, it was observed that a single dose GnRH agonist at the time of implantation improved the pregnancy rate in recipients after artificial endometrial preparation, inducing the downregulation of $\mathrm{GnRH}$ followed by a decrease in the levels of estrogen and P (8). In addition, GnRH agonist may directly influence the quality of the early embryo for the recipients without corpus luteum $(4,8)$, although a direct effect on the endometrium cannot be excluded. A previous in vitro study provided similar results, since GnRH agonist receptor was indicated to be broadly expressed in the human morula and at the blastocyst stage (9). The development of porcine and murine pre-implantation embryos is enhanced when incubated with GnRH agonist and diminished when incubated with GnRH antagonist $(10,11)$

In total, four previous systematic reviews concluded that there may be benefits from the addition of single-dose $\mathrm{GnRH}$ agonist to improve luteal support $(3,12-14)$, and it was suggested that this treatment is relatively safe and effective (15). However, it is required to perform a comprehensive and unbiased systematic review analyzing intensive studies and recent results (16). The aim of the present meta-analysis was to identify, analyze and summarize evidence from randomized controlled trials (RCTs) and examine the effects of single-dose GnRH agonist for luteal support in females undergoing IVF/ICSI cycles.

\section{Materials and methods}

Eligibility criteria. All published and ongoing RCTs assessing the administration effect of single-dose GnRH agonist during the luteal phase on IVF/ICSI outcomes were included in the present meta-analysis. Trials including egg donation and frozen embryo transfer cycles were excluded. Studies with multiple-dose GnRH agonist treatments in the luteal phase were excluded due to the lack of safety assessment and the large difference in GnRH agonist application protocols.

Grouping. In total, four groups were considered: i) Single-dose GnRH agonist administered as LPS at 5/6 days after IVF/ICSI procedures (GnRHa group); ii) regular support, where progesterone/estradiol/hCG was used for LPS (control group); iii) GnRH agonist was used to suppress premature LH surge (GnRH-a group); and iv) GnRH antagonist was used to suppress premature LH surge (GnRH-A group). In the GnRH-a group, a single GnRH agonist (Decapeptyl, 1.25-3.75 mg) was administered on day 24-26 of the cycle. After 2 weeks, Gn (Gonadotropin) are used to stimulate follicular development. In GnRH-A group, GnRH antagonist (Ganirelix acetate) was given $0.25 \mathrm{mg} / \mathrm{d}$ from day 5 or 6 of Gn administration until hCG day.

Outcome measures. The outcome measures used for the present meta-analysis were as follows: i) Ongoing pregnancy or live birth rate per transfer; ii) CPR per transfer; iii) multiple pregnancy rate; and iv) clinical abortion rate. Data in which the live birth rate was recorded was generally preferred. However, when live birth was not reported, data from an ongoing pregnancy (intrauterine live fetus with a gestational age $\geq 12$ weeks) were used as a measure for live birth, since the difference between live birth and ongoing pregnancy rates are limited and $<1 \%$ of pregnancies result in stillbirth $(17,18)$. When studies reported on clinical pregnancy and ongoing pregnancy without miscarriage rates, the number of clinical abortions was considered as being equal to the difference between the number of clinical pregnancies and ongoing pregnancies.

Search strategy. Published studies were searched in the following electronic databases: i) China National Knowledge Infrastructure (CNKI), ii) Wanfang database, iii) Chinese Biomedicine Literature Database (CBM), iv) Pubmed, v) EMBASE and vi) Cochrane Controlled Trials Register. The entries analyzed were published prior to June 1st, 2018. There was no language restriction. The following terms were used, adjusting for each database as necessary: 'Fertilization in vitro' OR 'in vitro fertilization' OR in vitro fertilizations' OR 'test-tube fertilization' OR 'fertilization, test-tube' OR 'fertilizations, test-tube' OR 'test tube fertilization' OR 'test-tube fertilizations' OR 'fertilizations in vitro' OR 'test-tube babies' OR 'babies, test-tube' OR 'baby, test-tube' OR 'test tube babies' OR 'intracytoplasmic sperm injection' OR 'IVF' OR 'ICSI' AND 'luteal support' OR 'luteal phase support' AND 'gonadotropin-releasing hormone agonist' OR 'GnRH-a' OR 'GnRHa' AND 'randomized controlled trial' OR 'randomized'.

Study selection and search results. The two researchers (MLS and CLL) independently screened the studies as follows: i) Duplicated articles were removed using NoteExpress software (version 3.2; Aegean Software Corp.); ii) two researchers read the titles and abstracts and manually removed the articles that did not meet the inclusion criteria of the present study; ii) the articles were further screened by reading the full text and excluded or included according to the inclusion and exclusion criteria of the present study. When inconsistent results were obtained, the issues were resolved by discussing with relevant experts in the field. Missing data were obtained from the authors of the respective studies whenever possible. In total, 468 records were retrieved in the initial electronic search: i) 92 records were from CNKI; ii) 70 records were from WANFANG; iii) 18 records were from CBM; iv) 65 records were from Pubmed; v) 112 records were from Embase; and vi) 111 records were from Cochrane. A total of 33 additional records were retrieved through manual search of potentially eligible studies and relevant reviews. A total of 501 records were assessed for eligibility. After removal of duplicate articles, 376 documents were retained and after reading the titles and abstracts, 318 records were removed. In addition, 38 articles that did not meet the inclusion criteria were excluded. Finally, among the 20 potentially relevant studies were obtained and a total of 8 studies fulfilled the inclusion criteria (19-26). A flowchart depicting the selection process is provided in Fig. 1.

Data extraction. In three studies $(20,22,24)$, the long GnRH-a ovarian treatment protocol starting in the mid-luteal phase of the preceding cycle was used, in four studies $(21,23,25,26)$, the GnRH-A multi low-dose ovarian treatment protocol was applied and in one study (19), both ovarian treatment protocol types were used. The trials published by Tesarik et al (8) used both the GnRH-a and the GnRH-A treatment protocol. In all studies, IVF or ICSI were performed. The characteristics of the eight studies identified, including the eligibility criteria, are provided in Table I. 


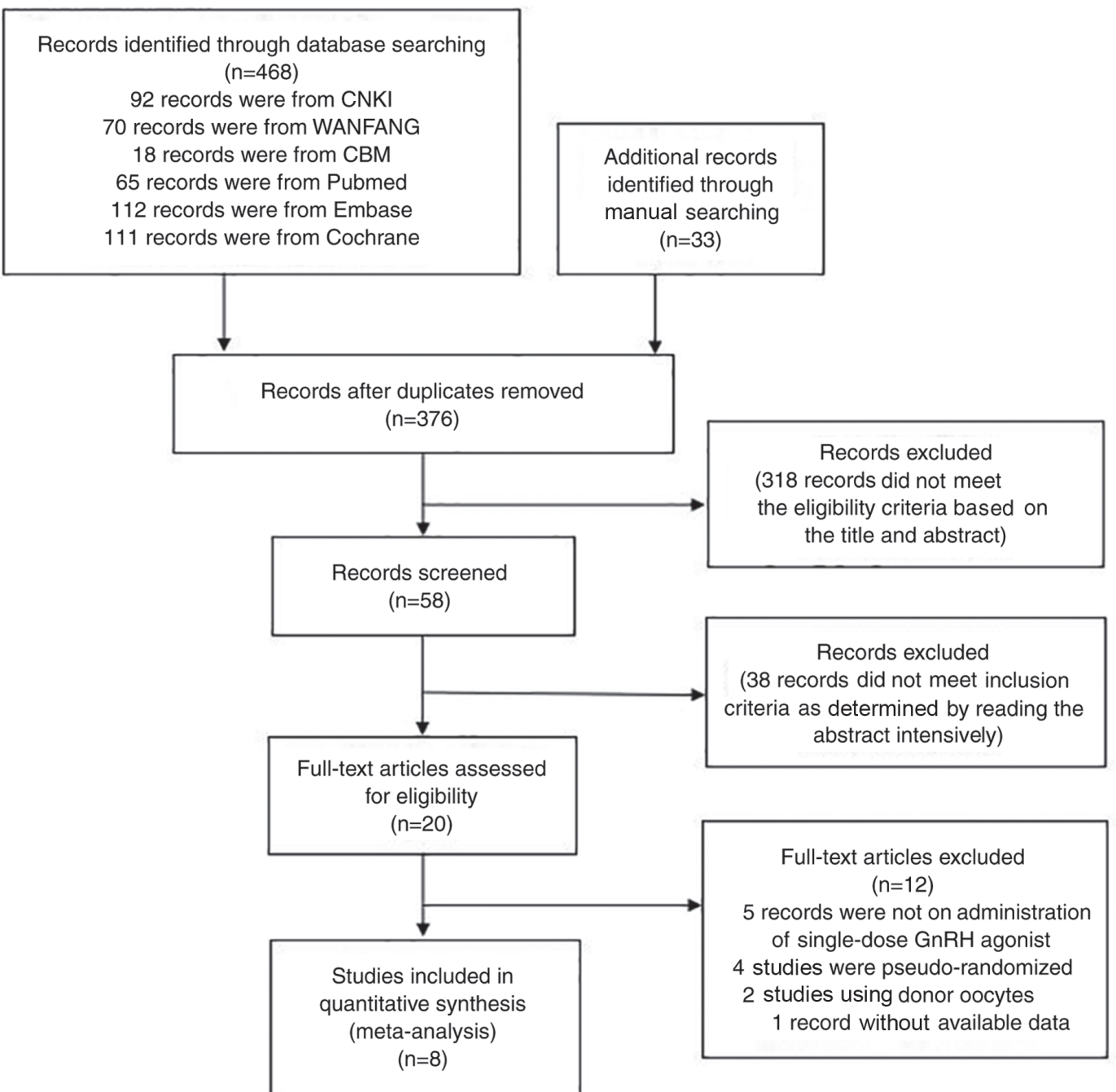

Figure 1. Flow diagram depicting the process of study selection. Published studies were searched in various electronic databases. All published and ongoing randomized controlled trials assessing the effect of single-dose GnRH agonist administration on in vitro fertilization/intracytoplasmic sperm injection outcomes were included. GnRH, gonadotropin-releasing hormone; CNKI, China National Knowledge Infrastructure; CBM, Chinese Biomedicine Literature Database.

Risk of bias within trials. The two researchers (MLS and CLL) evaluated the methodological quality of the studies included according to the RCT bias risk assessment tool recommended by the Cochrane systematic review guidelines (16). The evaluation included the following: i) Selection bias (random sequence generation and allocation concealment); ii) performance bias (blinding of participants and personnel); iii) detection bias (blinding of outcome assessment); iv) attrition bias (incomplete outcome data); v) reporting bias (selective reporting); and vi) other bias. In total, nine trials from eight studies were rated as 'low risk', 'unknown risk' or 'high risk'. In the case of disagreement, the two researchers discussed or resolved the issue by discussion with a third researcher $(\mathrm{RH})$.

Statistical analysis. The results for each of the studies eligible for the present meta-analysis were expressed as the risk ratio $(\mathrm{RR})$ and the precision of estimates were evaluated using the 95\% CI. The results of the previous studies were combined for the present meta-analysis using the DerSimonian and Laird method and a random-effects model was applied. The heterogeneity analysis was performed using the $\mathrm{I}^{2}$ test. When $\mathrm{P} \leq 0.05$ and $\mathrm{I}^{2} \geq 50 \%$, the studies were considered heterogeneous; $0 \leq \mathrm{I}^{2} \leq 25 \%$ indicated low heterogeneity, $25 \%<\mathrm{I}^{2} \leq 50 \%$ indicated moderate heterogeneity and $50 \%<\mathrm{I}^{2} \leq 75 \%$ indicated high heterogeneity. All results were combined for the meta-analysis using Revman 5.3 software (Cochrane Collaboration). In addition, sensitivity analysis and subgroup analysis were performed for the GnRH-a and GnRH-A groups according to a pituitary downregulation protocol, where the levels of FSH and LH are reduced. Sensitivity analysis was performed using a method that removed one document at a time to assess the impact of a single study on the results of the present meta-analysis. A re-analysis was performed after excluding the study identified as the source of heterogeneity.

\section{Results}

Ongoing pregnancy or live birth rate per transfer. In total, seven records from six studies were included to determine the effect of the treatments on ongoing pregnancy or live birth 


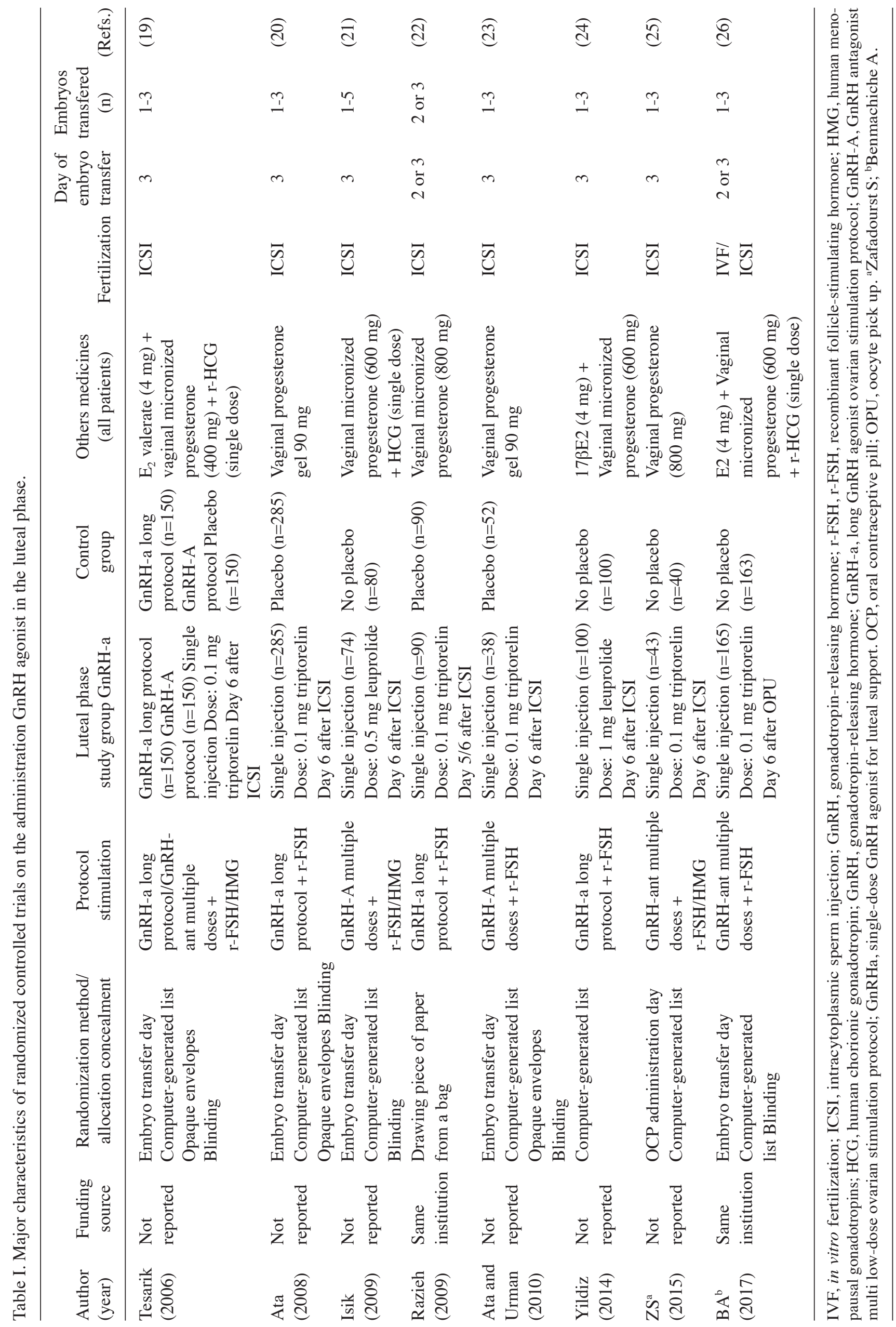




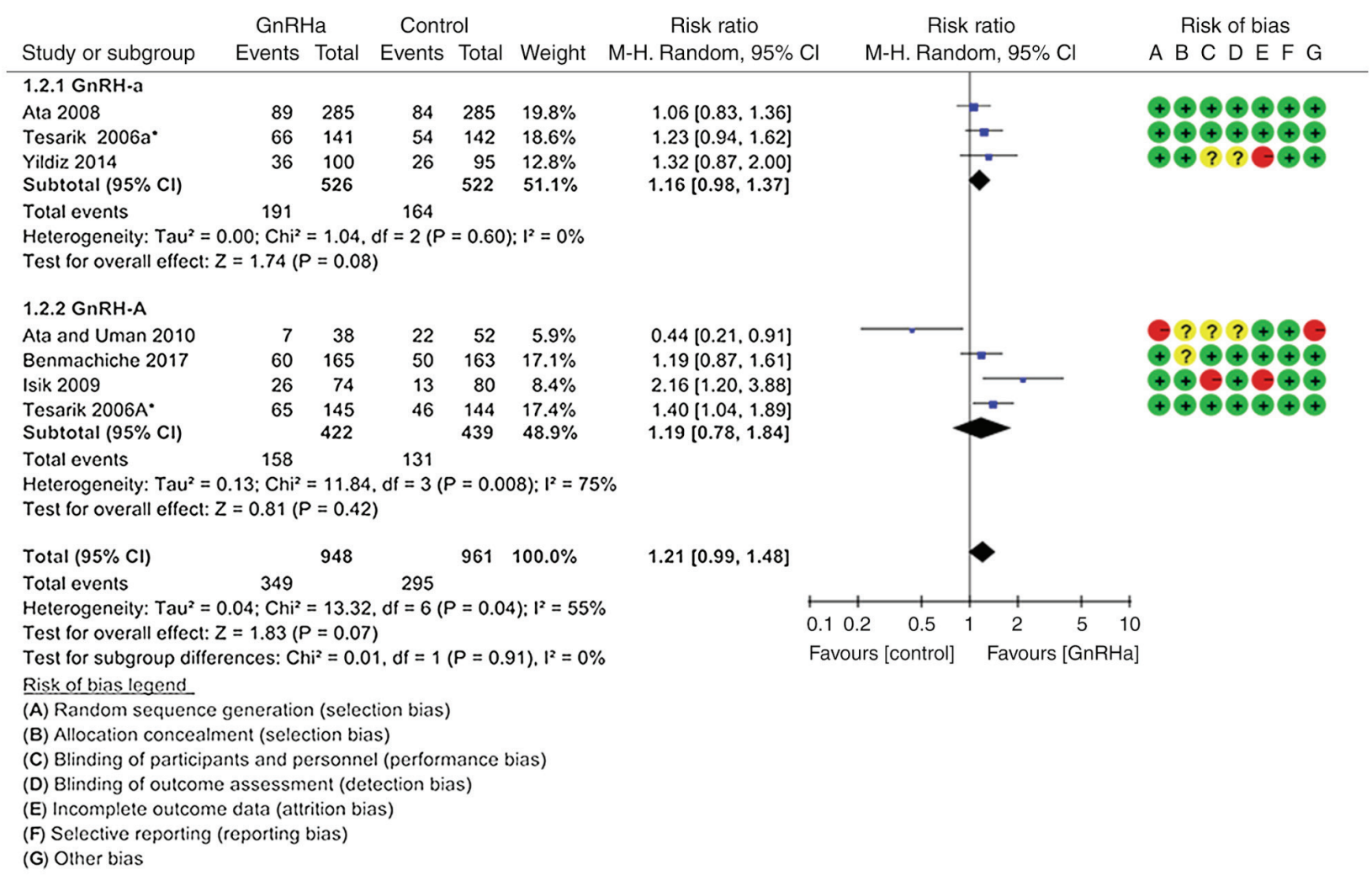

Figure 2. Forest plot for the meta-analysis on the influence of GnRHa on the ongoing pregnancy or live birth rate. In total, seven records from six studies were included. The pooled ongoing pregnancy or live birth rates per transfer were not significantly different between the GnRHa group (36.81\%; $349 / 948$ ) and the control group $(30.70 \% ; 295 / 961)$. Subgroup analysis according to GnRH agonists or antagonists used for luteinizing hormone suppression did not change the direction or the magnitude of the effect observed. GnRH, gonadotropin-releasing hormone; GnRH-a, long GnRH agonist ovarian treatment protocol; GnRH-A, GnRH antagonist ovarian treatment protocol; GnRHa, single-dose GnRH agonist for luteal support; Control, regular luteal support; MH, Mantel-Haentzel; df, degrees of freedom.

rate per transfer (Fig. 2) (19-21,23,24,26). The pooled ongoing pregnancy or live birth rate per transfer was not significantly different between the GnRHa group $(36.81 \%, 349 / 948)$ and the control group $(30.70 \%, 295 / 961 ; \mathrm{P}=0.070 ; \mathrm{RR}=1.21 ; 95 \%$ $\mathrm{CI}=0.99-1.48)$. Subgroup analysis according to the type of GnRH analogue used for LH suppression did not change the direction or the magnitude of the effect observed; there were no significant differences between the GnRH-a $(\mathrm{P}=0.080$; $\mathrm{RR}=1.16,95 \% \mathrm{CI}=0.98-1.37)(19,20,24)$ and $\mathrm{GnRH}-\mathrm{A}$ groups ( $\mathrm{P}=0.420 ; \mathrm{RR}=1.19,95 \% \mathrm{CI}=0.78-1.84$; Fig. 2) $(19,21,23,26)$.

However, there was high heterogeneity in the studies using a GnRH-A ovarian stimulation protocol $\left(\mathrm{P}=0.008 ; \mathrm{I}^{2}=75 \%\right)$. Therefore, sensitivity analysis under exclusion of the study by Ata and Urman (23), the source of heterogeneity, was performed (Fig. 3). The results suggested that the ongoing pregnancy or live birth rate per transfer was significantly higher in the GnRHa group $(37.5 \% ; 342 / 910)$ compared with that in the control group $(30.03 \% ; 273 / 909 ; \mathrm{P}=0.002$; $\mathrm{RR}=1.25,95 \% \mathrm{CI}=1.09-1.44)(19-21,24,26)$. This trend was more pronounced in the GnRH-A ovarian stimulation protocol ( $\mathrm{P}=0.010 ; \mathrm{RR}=1.41,95 \% \mathrm{CI}=1.08-1.84)(19,21,26)$. However, there were no significant differences in the $\mathrm{GnRH}$-a ovarian treatment protocol compared with control $(\mathrm{P}=0.080 ; \mathrm{RR}=1.16$, 95\% CI=0.98-1.37; Fig. 3) $(19,20,24)$.

CPR per transfer. In total, nine records from eight studies were included to calculate the CPR (Fig. 4) (19-26). The pooled CPR per transfer was significantly higher in the GnRHa group $(41.58 \% ; 437 / 1,051)$ compared with that in the control group $(33.64 \% ; 367 / 1,091 ; \mathrm{P}=0.010 ; \mathrm{RR}=1.28$; 95\% CI=1.06-1.55). There was significant heterogeneity in this comparison $\left(\mathrm{P}=0.020 ; \mathrm{I}^{2}=57 \%\right)$. However, in the subgroup of trials where the long GnRH-a ovarian stimulation protocol was used, the pooled CPR per transfer did not differ significantly between the GnRHa group $(41.72 \% ; 257 / 616)$ and the control group $(35.62 \% ; 218 / 612$; $\mathrm{P}=0.090 ; \mathrm{RR}=1.24,95 \% \mathrm{CI}=0.97-1.58)$, and the comparison did not exhibit significant heterogeneity $(\mathrm{P}=0.070$; $\left.\mathrm{I}^{2}=57 \%\right)(19,20,22,24)$. On the other hand, in the subgroup of trials where the GnRH-A ovarian stimulation protocol was used, the CPR per transfer was significantly superior in the GnRHa group $(41.38 \% ; 180 / 435)$ than in the control group (31.11\%; 149/479; $\mathrm{P}=0.040 ; \mathrm{RR}=1.32$, 95\% $\mathrm{CI}=0.96-1.81$; Fig. 4) $(19,21,23,25,26)$.

As above, a sensitivity analysis excluding the study by Ata and Urman (23) was performed (Fig. 5), and the pooled CPR per transfer was significantly higher in the GnRHa group $(41.95 \%$; $425 / 1,013)$ than that in the control group $(32.92 \% ; 342 / 1,039$; $\mathrm{P}=0.001 ; \mathrm{RR}=1.34,95 \% \mathrm{CI}=1.12-1.59)(19-22,24-26)$. This trend was more evident with the GnRH-A ovarian stimulation protocol $(\mathrm{P}<0.0001 ; \mathrm{RR}=1.45,95 \% \mathrm{CI}=1.20-1.74)(19,21,25,26)$. However, there were no significant differences in the GnRH-a ovarian stimulation protocol compared with control $(\mathrm{P}=0.090$; $\mathrm{RR}=1.24,95 \%$ CI=0.97-1.58; Fig. 5) $(19,20,22,24)$. 


\begin{tabular}{|c|c|c|c|c|c|c|c|c|c|}
\hline \multirow[b]{2}{*}{ Study or subgroup } & \multicolumn{2}{|c|}{ GnRHa } & \multicolumn{2}{|c|}{ Control } & \multirow[b]{2}{*}{ Weight } & Risk ratio & \multirow{2}{*}{\multicolumn{2}{|c|}{$\begin{array}{c}\text { Risk ratio } \\
\mathrm{M}-\mathrm{H} \text {. Random, } 95 \% \mathrm{Cl}\end{array}$}} & \multirow{2}{*}{$\begin{array}{l}\text { Risk of bias } \\
\text { A B C D F G }\end{array}$} \\
\hline & Events & Total & Events & Total & & M-H. Random, 95\% C & & & \\
\hline \multicolumn{10}{|l|}{ 1.2.1 GnRH-a } \\
\hline Ata 2008 & 89 & 285 & 84 & 285 & $25.4 \%$ & $1.06[0.83,1.36]$ & & 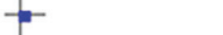 & \multirow{4}{*}{ 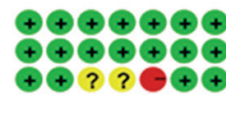 } \\
\hline Tesarik 2006a & 66 & 141 & 54 & 142 & $21.8 \%$ & $1.23[0.94,1.62]$ & & $=-$ & \\
\hline Yildiz 2014 & 36 & 100 & 26 & 95 & $10.4 \%$ & $1.32[0.87,2.00]$ & & 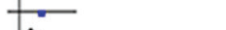 & \\
\hline Subtotal $(95 \% \mathrm{Cl})$ & & 526 & & 522 & $57.6 \%$ & $1.16[0.98,1.37]$ & & 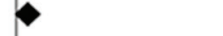 & \\
\hline Total events & 191 & & 164 & & & & & & \\
\hline \multicolumn{10}{|c|}{$\begin{array}{l}\text { Heterogeneity: } \text { Tau }^{2}=0.00: \mathrm{Chi}^{2}=1.04, \text { df }=2(P=0.60): 1^{2}=0 \% \\
\text { Test for overall effect: } Z=1.74(P=0.08)\end{array}$} \\
\hline \multicolumn{10}{|l|}{ 1.2.2 GnRH-A } \\
\hline Ata and Uman 2010 & 7 & 38 & 22 & 52 & & Not estimable & & & \multirow{5}{*}{ 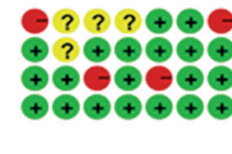 } \\
\hline Benmachiche 2017 & 60 & 165 & 50 & 163 & $18.1 \%$ & $1.19[0.87,1.61]$ & & - & \\
\hline Isik 2009 & 26 & 74 & 13 & 80 & $5.6 \%$ & $2.16[1.20,3.88]$ & & & \\
\hline Tesarik 2006A" & 65 & 145 & 46 & 144 & $18.8 \%$ & $1.40[1.04,1.89]$ & & & \\
\hline Subtotal $(95 \% \mathrm{Cl})$ & & 384 & & 387 & $42.4 \%$ & $1.41[1.08,1.84]$ & & 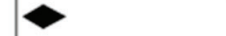 & \\
\hline Total events & 151 & & 109 & & & & & & \\
\hline \multicolumn{10}{|c|}{$\begin{array}{l}\text { Heterogeneity: } \mathrm{Tau}^{2}=0.02 ; \mathrm{Chi}^{2}=3.22 . \mathrm{df}=2(P=0.20): \mathrm{I}^{2}=38 \% \\
\text { Test for overall effect: } Z=2.50(P=0.01)\end{array}$} \\
\hline Total $(95 \% \mathrm{Cl})$ & & 910 & & 909 & $100.0 \%$ & $1.25[1.09,1.44]$ & & $\bullet$ & \\
\hline \multirow{2}{*}{\multicolumn{9}{|c|}{$\begin{array}{l}\text { Total events } \\
\text { Heterogeneity: } \text { Tau }^{2}=0.00: \mathrm{Chi}^{2}=5.81, \mathrm{df}=5(P=0.32): \mathrm{I}^{2}=14 \%\end{array}$}} & \\
\hline & & & & & & & $0.10 .2 \quad 0.5$ & $5 \quad 10$ & \\
\hline \multicolumn{7}{|c|}{ Test for subgroup differences: $\mathrm{Chi}^{2}=1.43, \mathrm{df}=1(P=0.23), \mathrm{I}^{2}=29.9 \%$} & Favours [control] & Favours [GnRHa] & \\
\hline \multicolumn{10}{|c|}{ Risk of bias legend } \\
\hline \multicolumn{10}{|c|}{ (A) Random sequence generation (selection bias) } \\
\hline \multicolumn{10}{|c|}{ (B) Allocation concealment (selection bias) } \\
\hline \multirow{2}{*}{\multicolumn{10}{|c|}{ (C) Blinding of participants and personnel (performance bias) }} \\
\hline \multirow{2}{*}{\multicolumn{9}{|c|}{ (D) Blinding of outcome assessment (detection bias) }} & \\
\hline \multicolumn{5}{|c|}{ (E) Incomplete outcome data (attrition bias) } & & & & & \\
\hline (F) Selective reporting & reporting & bias) & & & & & & & \\
\hline (G) Other bias & & & & & & & & & \\
\hline
\end{tabular}

Figure 3. Forest plot for the sensitivity analysis for ongoing pregnancy or live birth rate. Sensitivity analysis excluding the study by Ata and Urman was performed, and the results indicated that the pooled ongoing pregnancy or live birth rate per transfer in the GnRHa group was significantly higher than that in the control group. This trend was more significant following GnRH-A ovarian stimulation protocol. GnRH, gonadotropin-releasing hormone; GnRH-a, long GnRH agonist ovarian stimulation protocol; GnRH-A, GnRH antagonist multi low-dose ovarian stimulation protocol; GnRHa, single-dose GnRH agonist for luteal support; Control, regular luteal support; MH, Mantel-Haentzel; df, degrees of freedom.

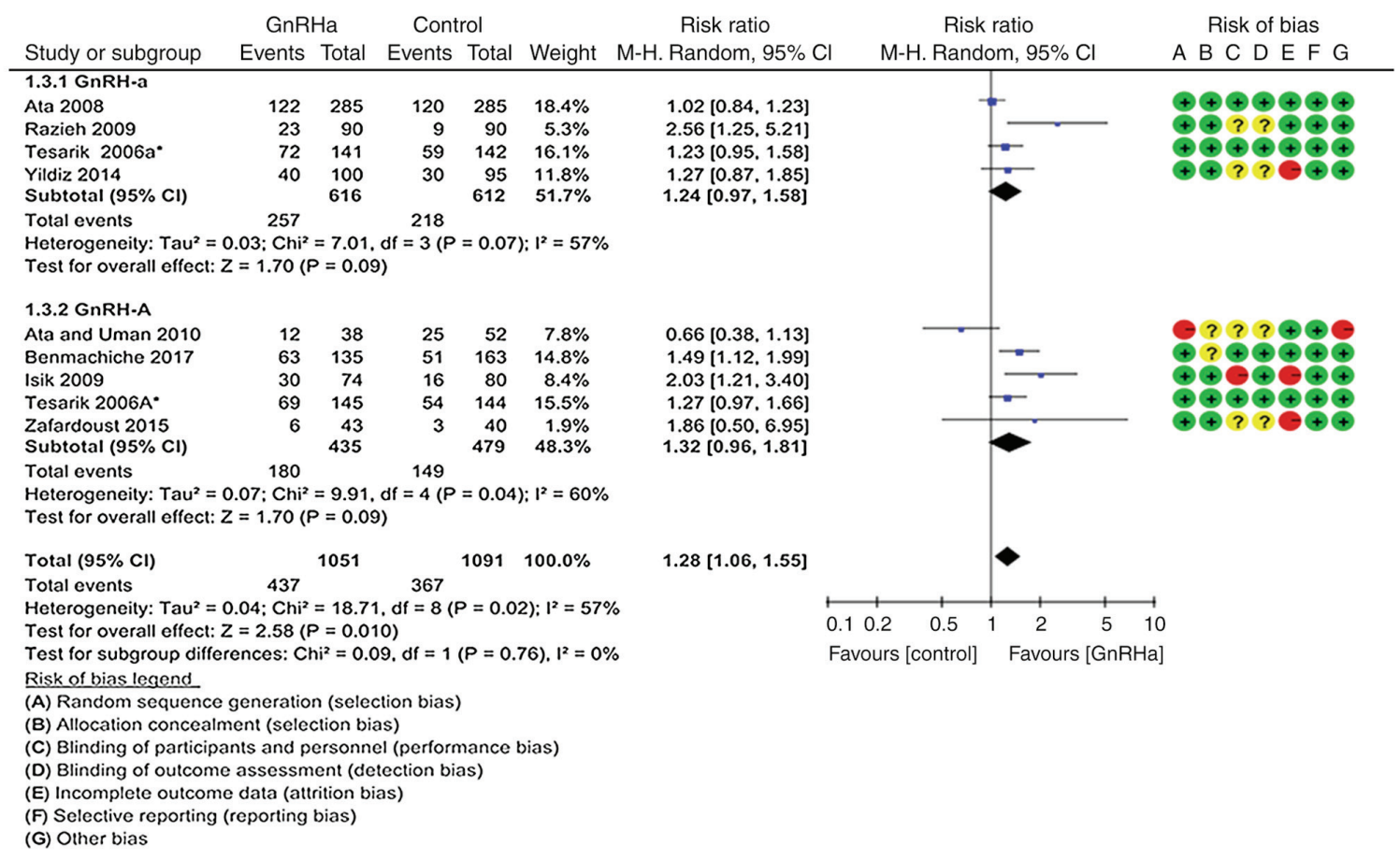

Figure 4. Forest plot for the CPR. In total, nine records from eight studies were included. The pooled CPR per transfer rate was significantly higher in the GnRHa group than in the control group. There was heterogeneity in this comparison. However, in the subgroup of trials using the long GnRH-a ovarian stimulation protocol, the pooled CPR per transfer did not differ significantly between the GnRHa group and the control group (35.62\%; 218/612). Furthermore, this comparison exhibited a certain heterogeneity $\left(\mathrm{P}=0.070 ; \mathrm{I}^{2}=57 \%\right)(19,20,22,24)$. By contrast, in the subgroup of trials using the GnRH-A ovarian stimulation protocol, the CPR per transfer was significantly different in the GnRHa group compared with that in the control group. CPR, clinical pregnancy rate; GnRH, gonadotropin-releasing hormone; GnRH-a, long GnRH agonist ovarian stimulation protocol; GnRH-A, GnRH antagonist multi low-dose ovarian stimulation protocol; GnRHa, single-dose GnRH agonist for luteal support; Control: Regular luteal support; MH, Mantel-Haentzel; df, degrees of freedom. 


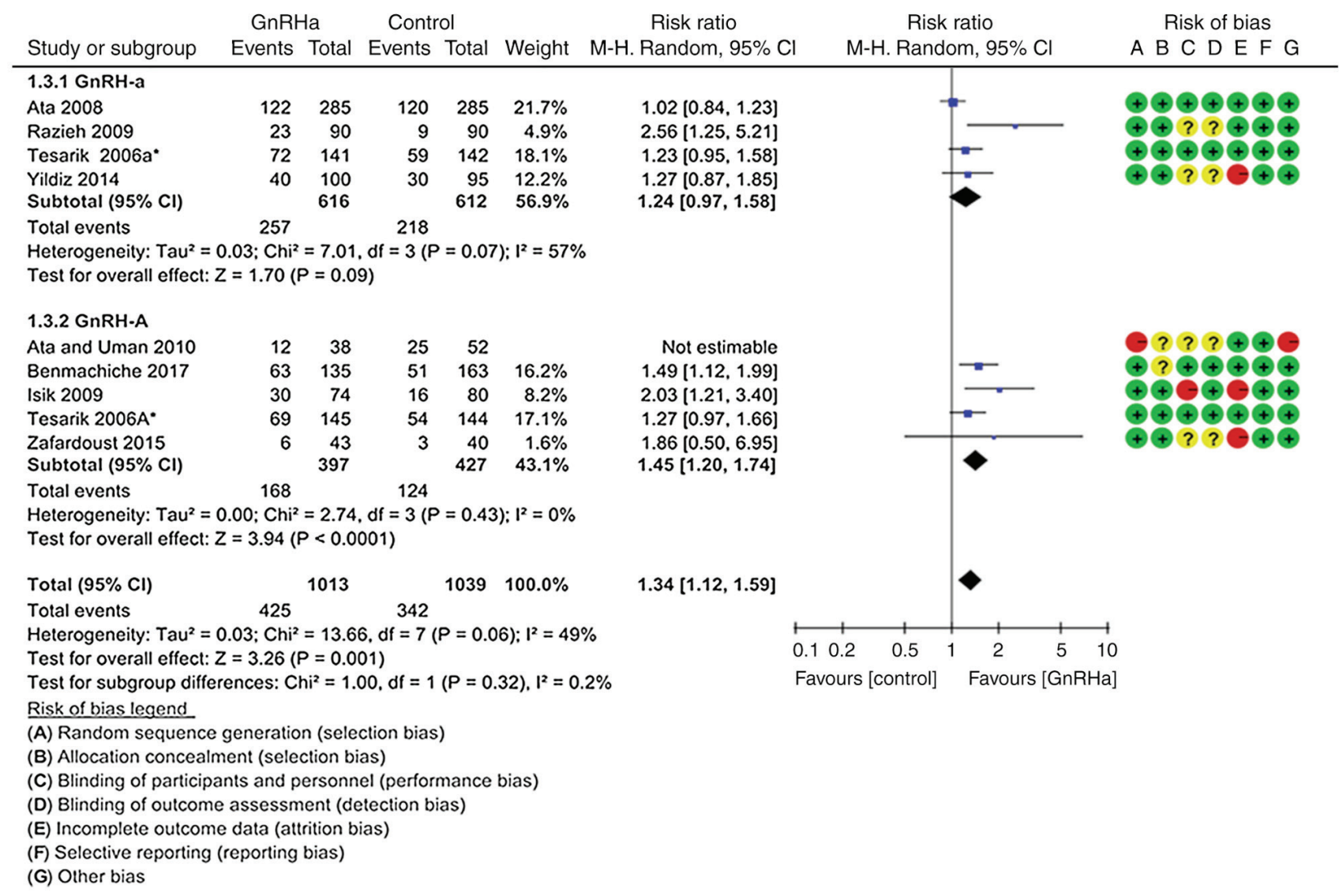

Figure 5. Forest plot for the sensitivity analysis for the CPR. Sensitivity analysis excluding the study by Ata and Urman was performed, and the results suggested that the pooled CPR per transfer was significantly higher in the GnRHa group compared with that in the control group. This trend was more significant following the GnRH-A ovarian stimulation protocol. However, there were no significant differences in the GnRH-a ovarian stimulation protocol compared with the control group. CPR, clinical pregnancy rate; $\mathrm{GnRH}$, gonadotropin-releasing hormone; GnRH-a, long GnRH agonist ovarian stimulation protocol; GnRH-A, GnRH antagonist multi low-dose ovarian stimulation protocol; GnRHa, single-dose GnRH agonist for luteal support; Control, regular luteal support; MH, Mantel-Haentzel; df, degrees of freedom.

Multiple pregnancy rate per pregnancy. As indicated in Fig. 6, the rate of multiple pregnancy per pregnancy was significantly higher in the GnRHa group (32.82\%; 106/323) compared with that in the control group $(19.17 \%$; 51/266; $\mathrm{P}=0.020 ; \mathrm{RR}=2.37,95 \% \mathrm{CI}=1.17-4.81)$. In total, four studies were included (19-21,24). In the subgroup of trials where the GnRH-A ovarian stimulation protocol was used, the multiple pregnancy rate per pregnancy was significantly higher in the GnRH-A group $(33.68 \% ; 32 / 95)$ than that in the control group $(8.06 \% ; 5 / 62 ; \mathrm{P}=0.003 ; \mathrm{RR}=3.70 ; 95 \% \mathrm{CI}=1.57-8.69)(19,21)$. However, the difference between the GnRH-a ovarian stimulation protocol and the control group was not significant $(\mathrm{P}=0.140 ; \mathrm{RR}=1.88 ; 95 \% \mathrm{CI}=0.82-4.34$; Fig. 6) $(19,20,24)$.

Clinical abortion rate. As indicated in Fig. 7, the clinical abortion rate was not significantly different between the GnRHa group $(14.46 \% ; 59 / 408)$ and the control group $(16.90 \% ; 60 / 355$; $\mathrm{P}=0.790 ; \mathrm{RR}=0.94 ; 95 \% \mathrm{CI}=0.61-1.45)$. These results were obtained from six studies $(19-21,23,24,26)$. There were also no significant differences in the subgroup analyses.

According to the sensitivity analysis excluding the study by Ata and Urman (23), the clinical abortion rates were not significantly different between the GnRHa group and the control group $\left(\mathrm{P}=0.310 ; \mathrm{RR}=0.84,95 \% \mathrm{CI}=0.61-1.17 ; \mathrm{I}^{2}=0 \%\right.$;
Fig. 8). In addition, the subgroup analyses did not provide any significant differences.

Risk of bias of the individual studies are presented in the forest plots shown in Figs. 2-8. There was 'high risk' and high heterogeneity in the study by Ata and Urman (23). The high risk of this study mainly comes from selection bias (random sequence generation and allocation concealment) and other bias.

\section{Discussion}

In 1993, Wilshire et al (27) reported that the use of GnRH agonist during early pregnancy did not have any adverse effect on pregnancy outcomes. Over the past years, the question regarding whether GnRH agonist may be used during LPS to improve pregnancy outcomes has attracted increasing attention. Zafardoust et al (25) indicated that subcutaneous injection of $0.1 \mathrm{mg}$ triptorelin on the 6th day after oocyte collection increased the rate of embryo implantation and pregnancy rate in ICSI patients who were downregulated following GnRH antagonist regimen. A previous study identified increased levels of LH, as well as increased embryo implantation and CPRs, following intranasal inhalation of buserelin as LPS treatment compared with those following vaginal administration 


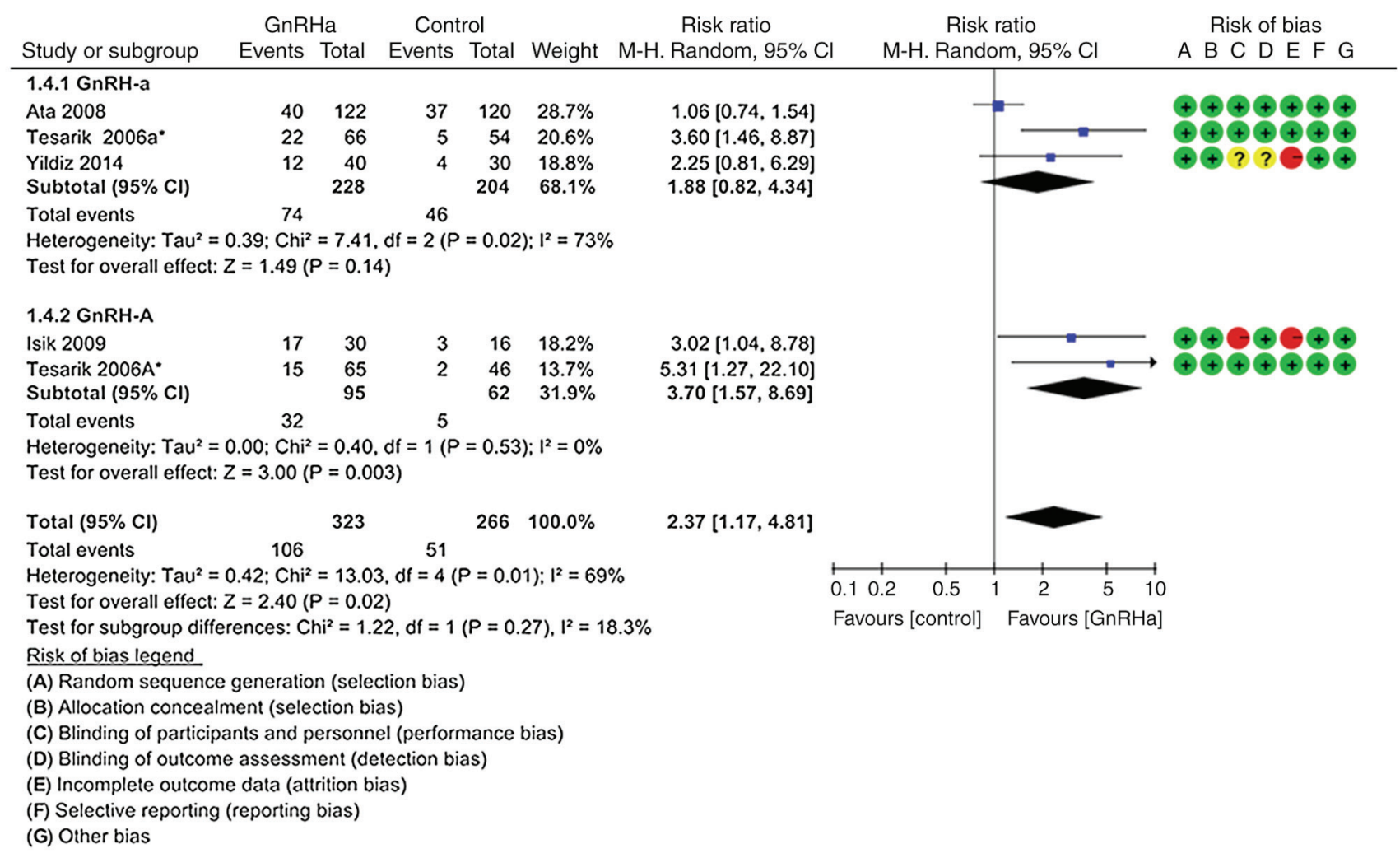

Figure 6. Forest plot for the multiple pregnancy rate. The probability of multiple pregnancy per pregnancy was significantly higher in the GnRHa group compared with that in the control group. In total, four studies were included. In the subgroup of trials using the GnRH-A ovarian stimulation protocol, the multiple pregnancy rate was higher in the GnRHa group than in the control group. However, there were no significant differences in the GnRH-a ovarian stimulation protocol. GnRH, gonadotropin-releasing hormone; GnRH-a, long GnRH agonist ovarian stimulation protocol; GnRH-A, GnRH antagonist multi low-dose ovarian stimulation protocol; GnRHa, single-dose GnRH agonist for luteal support; Control, regular luteal support; MH, Mantel-Haentzel; df, degrees of freedom.

of progesterone, but the differences were not statistically significant (28). However, Aboulghar et al (29) indicated that continuous injection of GnRH agonist at $0.1 \mathrm{mg} /$ day from the luteal phase to hCG test day (14 days after embryo transfer) was not sufficient to increase the rate of ongoing pregnancy and CPR, and exhibited a negative effect; therefore, the effect of GnRH treatment in LPS remains controversial.

The studies included were single-center RCTs with a small sample size, and the present study included nine records from eight studies with a total of 2,142 embryo transfer cycles. The present results suggested that addition of GnRH agonist during LPS significantly increased the ongoing pregnancy or live birth rate per transfer, CPR per transfer and multiple pregnancy rate per pregnancy. The present meta-analysis suggested that the majority of the present results exhibited high heterogeneity. Therefore, a sensitivity analysis was performed using Revman 5.3 software, and it was indicated that the high heterogeneity was primarily caused by the study by Ata and Urman (23). Sensitivity analysis was performed following the exclusion of this study, resulting in significantly higher rates of ongoing pregnancy or live birth per transfer $(\mathrm{P}=0.002)$, clinical pregnancy per transfer $(\mathrm{P}=0.001)$ and multiple pregnancy per pregnancy $(\mathrm{P}=0.020)$ in the $\mathrm{GnRHa}$ group compared with those in the control group. In addition, meta-analysis was performed for subgroups of trials that had used a long GnRH-a ovarian stimulation protocol, indicating that ongoing pregnancy or live birth rate per transfer $(\mathrm{P}=0.080), \mathrm{CPR}$ per transfer $(\mathrm{P}=0.090)$ and multiple pregnancy rate per pregnancy $(\mathrm{P}=0.140)$ were not significantly different between the two groups. Furthermore, the results from trials that had used GnRH-A multi-dose ovarian stimulation protocols indicated significantly higher ongoing pregnancy or live birth rate per transfer $(\mathrm{P}=0.010), \mathrm{CPR}$ per transfer $(\mathrm{P}<0.0001)$ and multiple pregnancy rate per pregnancy $(\mathrm{P}=0.003)$ compared with those in the control group. Therefore, the present results suggested that the addition of single-dose GnRH agonist during LPS was clinically beneficial for pregnancy outcomes.

At present, the mechanisms underlying the use of GnRH agonist to improve pregnancy outcomes remain to be fully elucidated. Previous studies have suggested various possible mechanisms of action of GnRH agonist. GnRH agonist may act on the pituitary gland or ovary during the luteal phase (30-32); GnRH agonist may stimulate the ovaries to produce estrogen and $\mathrm{P}$ by stimulating the pituitary to produce $\mathrm{LH}$, and GnRH agonist may produce estrogen and $\mathrm{P}$ by acting directly on the corpus luteum (20). Furthermore, GnRH agonist may directly act on the embryo and placenta during implantation (8); GnRH agonist promotes the expression of $\mathrm{GnRH}$ agonist receptor in placental cytotrophoblasts and syncytiotrophoblasts and increases serum hCG levels by upregulating $\mathrm{GnRH}$ agonist receptor levels and stimulating placental production of hCG, thus improving the implantation ability of the embryo $(33,34)$. In addition, GnRH agonist promoted the growth of mouse embryos when it was added in the culture medium; however, 


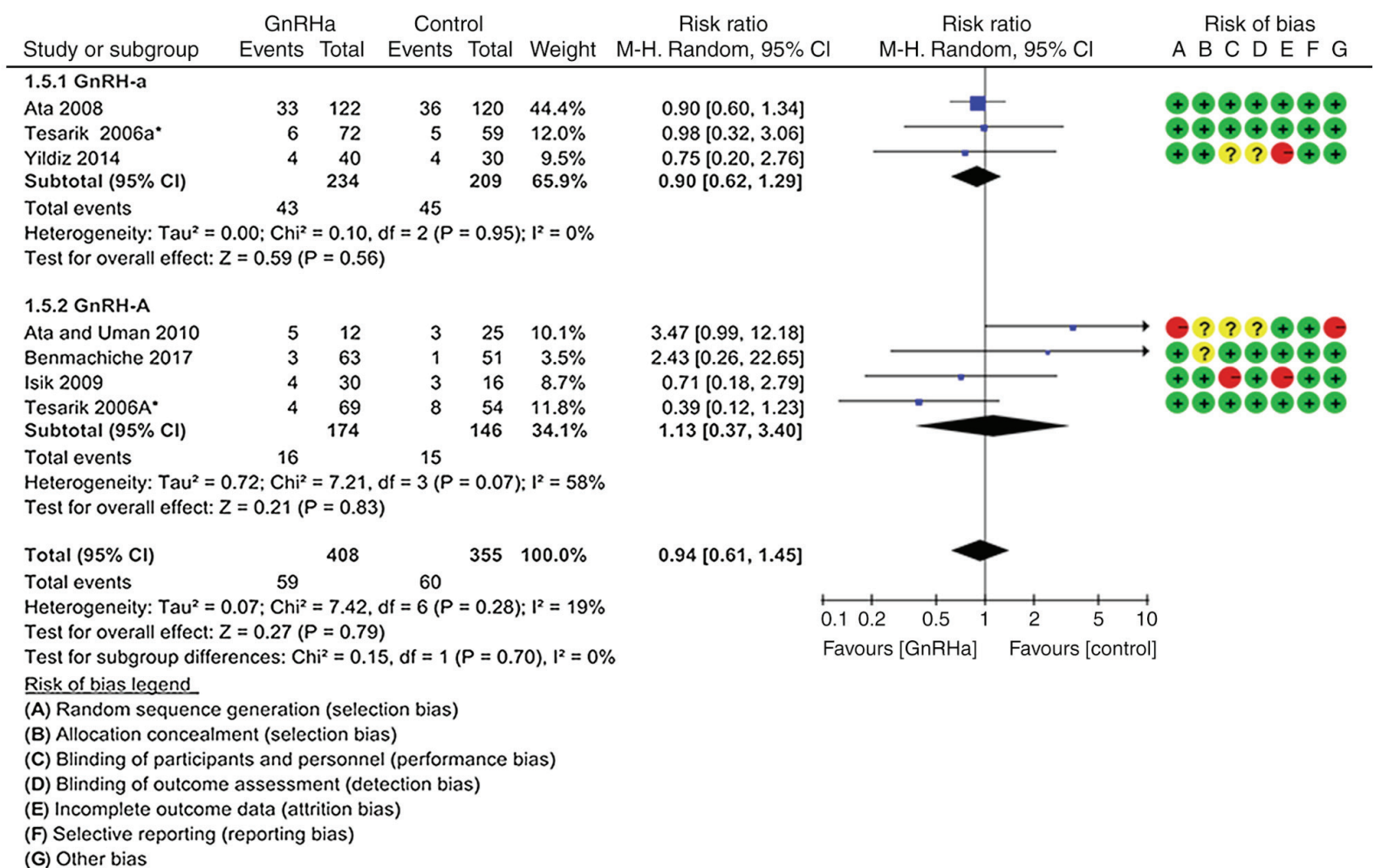

Figure 7. Forest plot for the clinical abortion rate. The clinical abortion rate was not significantly different between the GnRHa group and the control group. The results were derived from six studies. GnRH, gonadotropin-releasing hormone; GnRH-a, long GnRH agonist ovarian stimulation protocol; GnRH-A, GnRH antagonist multi low-dose ovarian stimulation protocol; GnRHa, single-dose GnRH agonist for luteal support; Control, regular luteal support; MH, Mantel-Haentzel; df, degrees of freedom.

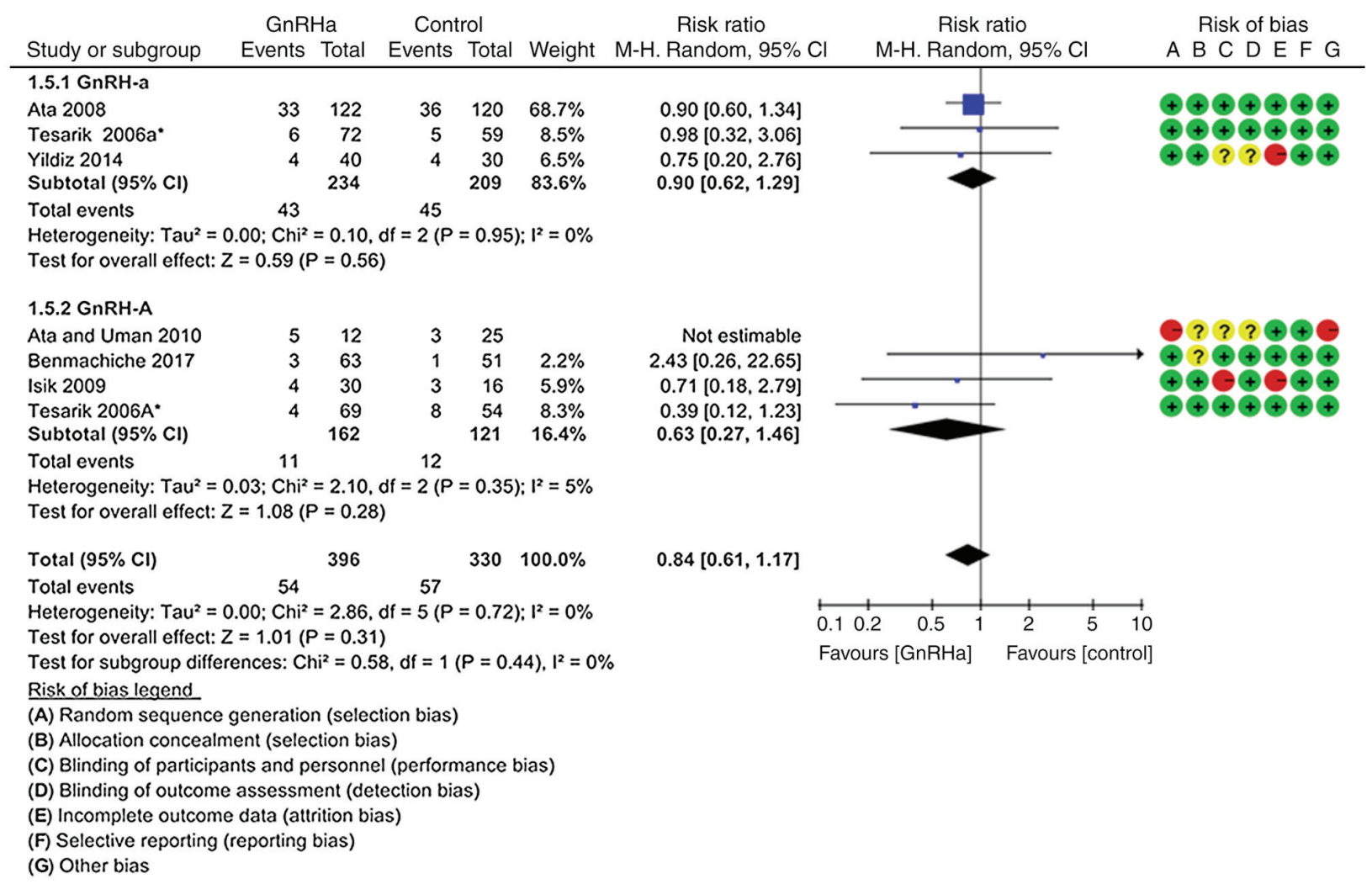

Figure 8. Forest plot for the sensitivity analysis for the clinical abortion rate. Sensitivity analysis excluding the study by Ata and Urman comprised five studies, where no significance was observed. GnRH, gonadotropin-releasing hormone; GnRH-a, long GnRH agonist ovarian stimulation protocol; GnRH-A, GnRH antagonist multi low-dose ovarian stimulation protocol; GnRHa, single-dose GnRH agonist for luteal support; Control, regular luteal support; MH, Mantel-Haentzel; df, degrees of freedom. 
GnRH antagonist had a detrimental effect on mouse embryos (11). Therefore, it is possible that pre-implantation mouse embryos may express the GnRH agonist receptor. Furthermore, GnRH agonist may directly act via the GnRH agonist receptor on the surface of the endometrium $(8,9)$.

The major limitation of the present meta-analysis is that there may be publication bias in the studies analyzed, and this may be due to a number of reasons; the present analysis only included studies including full texts, and abstract-only papers were excluded due to the lack of complete statistical data. In addition, three of articles included in the meta-analysis $(21,24,25)$ exhibited a high risk of bias. In addition, the evidence is limited to recommend the use of GnRHa in the luteal phase support. Therefore, multi-center randomized controlled studies following a unified standardized scheme are required.

In conclusion, the present meta-analysis study suggested that administration of single-dose GnRH agonist for LPS in females partaking in IVF/ICSI was able to increase the ongoing pregnancy or live birth rate per transfer, CPR per transfer and multiple pregnancy rate per pregnancy. However, GnRH agonist did not affect the clinical abortion rate. In addition, since the multiple pregnancy risk increased significantly, GnRH agonist administration may be a better option for LPS with single embryo transfer. Therefore, GnRH agonist treatment may be an ideal choice for LPS in patients undergoing IVF/ICSI. However, further RCTs or multi-center randomized controlled studies are required prior to clinical application of GnRH agonist.

\section{Acknowledgements}

Not applicable.

\section{Funding}

The present study was supported by the Natural Science Foundation of Ningxia (grant no. NZ17180).

\section{Availability of data and materials}

All data generated or analyzed during the present study are included in this published article.

\section{Authors' contributions}

MLS conceived the study and was a major contributor in writing the manuscript. CLL participated in the design and coordination of the study and helped to draft the manuscript. $\mathrm{RH}$ performed the statistical analysis. FMW and $\mathrm{ZHH}$ contributed to the collection, analysis of data and edited the manuscript. All authors have read and approved the final manuscript.

\section{Ethics approval and consent to participate}

Not applicable.

\section{Patient consent for publication}

Not applicable.

\section{Competing interests}

The authors declare that they have no competing interests.

\section{References}

1. Tavaniotou A, Albano C, Smitz J and Devroey P: Impact ofovarian stimulation on corpus luteum function and embryonicimplantation. J Reprod Immunol 55: 123-130, 2002.

2. Pritts EA and Atwood AK: Luteal phase support in infertilitytreatment: A meta-analysis of the randomized trials. Hum Reprod 17: 2287-2299, 2002.

3. van der Linden M, Buckingham K, Farquhar C, Kremer JA and Metwally M: Luteal phase support for assisted reproduction cycles. Cochrane Database Syst Rev: CD009154, 2015.

4. Fatemi HM: The luteal phase after 3 decades of IVF: What do we know? Reprod Biomed Online 19 (Suppl 4): S4331, 2009.

5. Devroey P, Bourgain C, Macklon NS and Fauser BC: Reproductive biology and IVF: Ovarian stimulationand endometrial receptivity. Trends Endocrinol Metab 15: 84-90, 2004.

6. Gizzo S, Andrisani A, Esposito F, Noventa M, Di Gangi S, Angioni S, Litta P, Gangemi M and Nardelli GB: Which luteal phase support is better for each IVF stimulation protocol to achieve the highest pregnancy rate? A superiority randomized clinical trial. Gynecol Endocrinol 30: 902-908, 2014.

7. Fujii S, Sato S, Fukui A, Kimura H, Kasai G and Saito Y: Continuous administration of gonadotrophin-releasing hormone agonist during the luteal phase in IVF. Hum Reprod 16: 1671-1675, 2001.

8. Tesarik J, Hazout A and Mendoza C: Enhancement of embryo developmental potential by a single administration of $\mathrm{GnRH}$ agonist at the time of implantation. Hum Reprod 19: 1176-1180, 2004.

9. Casan EM, Raga F and Polan ML: GnRH mRNA and protein expression in human preimplantation embryos. Mol Hum Reprod 5: 234-239, 1999.

10. Nam DH, Lee SH, Kim HS, Lee GS, Jeong YW, Kim S, Kim JH, Kang SK, Lee BC and Hwang WS: The role of gonadotropin-releasing hormone $(\mathrm{GnRH})$ and its receptor in development of porcine preimplantation embryos derived from in vitro fertilization. Theriogenology 63: 190-201, 2005.

11. Raga F, Casan EM, Kruessel J, Wen Y, Bonilla-Musoles F and Polan ML: The role of gonadotropin- releasing hormone in murine preimplantation embryonic development. Endocrinology 140: 3705-3712, 1999.

12. Kyrou D, Kolibianakis EM, Fatemi HM, Tarlatzi TB, Devroey P and Tarlatzis BC: Increased live birth rates with $\mathrm{GnRH}$ agonist addition for luteal support in ICSI/IVF cycles: A systematic review and meta-analysis. Hum Reprod Update 17: 734-740, 2011.

13. Oliveira JB, Baruffi R, Petersen CG, Mauri AL, Cavagna M and Franco JG Jr: Administration of single-dose GnRH agonist in the luteal phase in ICSI cycles: A meta-analysis. Reprod Biol Endocrinol 8: 107, 2010.

14. Yu LP, Liu N and Liu Y: Effect of luteal-phase gonadotropin-releasing hormone agonist administration on pregnancy outcome in IVF/ICSI cycles: A systematic review and Meta-analysis. Zhonghua Fu Chan Ke Za Zhi 51: 850-858, 2016 (In Chinese).

15. Zhou W, Zhuang Y, Pan Y and Xia F: Effects and safety of GnRH-a as a luteal support in women undertaking assisted reproductive technology procedures: Follow-up results for pregnancy, delivery, and neonates. Arch Gynecol Obstet 295: 1269-1275, 2017.

16. Martins WP, Ferriani RA, Navarro PA and Nastri CO: GnRH agonist during luteal phase for women undergoing assisted reproductive techniques: Systematic review and meta-analysis of randomized controlled trials. Ultrasound Obstet Gynecol 47: 144-151, 2016.

17. Gordon A, Raynes-Greenow C, McGeechan K, Morris J and Jeffery H: Risk factors for antepartum stillbirth and the influence of maternal age in New South Wales Australia: A population based study. BMC Pregnancy Childbirth 13: 12, 2013.

18. Reddy UM, Laughon SK, Sun L, Troendle J, Willinger M and Zhang J: Prepregnancy risk factors for antepartum stillbirth in the United States. Obstet Gynecol 116: 1119-1126, 2010. 
19. Tesarik J, Hazout A, Mendoza-Tesarik R, Mendoza N and Mendoza C: Beneficial effect of luteal-phase GnRH agonist administration on embryo implantation after ICSI in both GnRH agonist- and antagonist-treated ovarian stimulation cycles. Hum Reprod 21: 2572-2579, 2006.

20. Ata B, Yakin K, Balaban B and Urman B: GnRH agonist protocol administration in the luteal phase in ICSI-ET cycles stimulated with the long $\mathrm{GnRH}$ agonist protocol: A randomized, controlled double blind study. Hum Reprod 23: 668-673, 2008.

21. Isik AZ, Caglar GS, Sozen E, Akarsu C, Tuncay G, Ozbicer T and Vicdan K: Single-dose GnRH agonist administration in the luteal phase of GnRH antagonist cycles: A prospective randomized study. Reprod Biomed Online 19: 472-477, 2009.

22. Razieh DF, Maryam AR and Nasim T: Beneficial effect of luteal-phase gonadotropin-releasing hormone agonist administration on implantation rate after intracytoplasmic sperm injection. Taiwan J Obstet Gynecol 48: 245-248, 2009.

23. Ata B and Urman B: Single dose GnRH agonist administration in the luteal phase of assisted reproduction cycles: Is the effect dependent on the type of GnRH analogue used for pituitary suppression? Reprod Biomed Online 20: 165-166, 2010.

24. Yildiz GA, Şükür YE, Ateş C and Aytaç R: The addition of gonadotrophin releasing hormone agonist to routine luteal phase support in intracytoplasmic sperm injection and embryo transfer cycles: A randomized clinical trial. Eur J Obstet Gynecol Reprod Biol 182: 66-70, 2014.

25. Zafardoust S, Jeddi-Tehrani M, Akhondi MM, Sadeghi MR, Kamali K, Mokhtar S, Badehnoosh B, Arjmand-Teymouri F, Fatemi $\mathrm{F}$ and Mohammadzadeh A: Effect of administration of single dose GnRH agonist in luteal phase on outcome of ICSI-ET cycles in women with previous history of IVF/ICSI failure: A randomized controlled trial. J Reprod Infertili 16: 116-120, 2015.

26. Benmachiche A, Benbouhedja S, Zoghmar A, Boularak A and Humaidan P: Impact of mid-luteal phase GnRH agonist administration on reproductive outcomes in GnRH agonist-triggered cycles: A randomized controlled trial. Front Endocrinol (Lausanne) 8: 124, 2017.
27. Wilshire GB, Emmi AM, Gagliardi CC and Weiss G: Gonadotropin-releasing hormone agonist administration in early human pregnancy is associated with normal outcomes. Fertil Steril 60: 980-983, 1993.

28. Pirard C,Loumaye E, Laurent Pand Wyns C: Contribution to more patient-friendly ART treatment: Efficacy of continuous low-dose $\mathrm{GnRH}$ agonist as the only luteal support-results of a prospective, randomized, comparative study. Int J Endocrinol 2015: 727569, 2015.

29. Aboulghar MA, Marie H, Amin YM, Aboulghar MM, Nasr A, Serour GI and Mansour RT: GnRH agonist plus vaginal progesterone for luteal phase support in ICSI cycles: A randomized study. Reprod Biomed Online 30: 52-56, 2015.

30. Chillik CF, Itskovitz J, Hahn DW, McGuire GL, Danforth DR and Hodgen GD: Characterizing pituitary response to a gonadotropin-releasing hormone $(\mathrm{GnRH})$ antagonist in monkeys: Tonic follicle-stimulating hormone/luteinizing hormone secretion versus acute $\mathrm{GnRH}$ challenge tests before, during, and after treatment. Fertil Steril 48: 480-485, 1987.

31. Gordon K, Williams RF, Danforth DR and Hodgen GD: A novel regimen of gonadotropin-releasing hormone $(\mathrm{GnRH})$ antagonist plus pulsatile GnRH: Controlled restoration of gonadotropin secretion and ovulation induction. Fertil Steril 54: 1140-1145, 1990.

32. Felberbaum RE, Reissmann T, Küpker W, Bauera O, al Hasania S, Diedrich $\mathrm{C}$ and Diedricha K: Preserved pituitary response under ovarian stimulation with HMG and GnRH antagonists (Cetrorelix) in women with tubal infertility. Eur J Obstet Gynecol Reprod Biol 61: 151-155, 1995.

33. Iwashita M, Kudo Y, Shinozaki Y and Takeda Y: Gonadotropin-releasing hormone increases serum human chorionic gonadotropin in pregnant women. Endocr J 40: 539-544, 1993.

34. Lin LS, Roberts VJ and Yen SS: Expression of human gonadotropin-releasing hormone receptor gene in the placenta and its functional relationship to human chorionic gonadotropin secretion. J Clin Endocrinol Metab 80: 580-585, 1995. 